\title{
Simulation and Optimization of DPP Hydrodynamics and Radiation Transport for EUV Lithography Devices
}

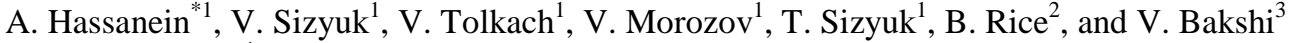 \\ ${ }^{1}$ Argonne National Laboratory, Argonne, IL 60439, USA \\ ${ }^{2}$ Intel Corporation, Component Research Division, Hillsboro, OR 97124, USA \\ ${ }^{3}$ International Sematech, Austin, TX 78741, USA
}

\begin{abstract}
Discharge produced plasma (DPP) devices are being used as a light source for Extreme Ultraviolet (EUV) Lithography. A key challenge for DPP is achieving sufficient brightness to support the throughput requirements of exposure tools for high-volume manufacturing lithography. An integrated model is being developed to simulate the environment of the EUV source and optimize the output of the source. The model describes the hydrodynamic and optical processes that occur in DPP devices. It takes into account plasma evolution and magnetohydrodynamic processes as well as detailed photon radiation transport. The total variation diminishing scheme in the Lax-Friedrich formulation for the description of magnetic compression and diffusion in a cylindrical geometry is used. Several models are being developed for opacity calculations: a collisional radiation equilibrium model, a self-consistent field model with Auger processes, and a non-stationary kinetic model. Radiation transport for both continuum and lines with detailed spectral profiles is taken into account. The developed models are being integrated into the HEIGHTS-EUV computer simulation package. Preliminary results of a numerical simulation of xenon gas hydrodynamics and EUV radiation output are presented for various plasma conditions.
\end{abstract}

Keywords: Discharge-produced plasma, HEIGHTS-EUV, MHD, radiation transport, numerical simulation

\section{INTRODUCTION}

This paper presents an integrated model that is being developed to describe the hydrodynamics and optical processes that occur in discharge-produced plasma (DPP) devices. The developed model will eventually address three main subjects: plasma evolution and magnetohydrodynamic (MHD) processes, detailed photon radiation transport, and interaction between plasma/radiation and material. Regions with differing propagation speeds of perturbation require accurate numerical solutions of the MHD equations. The total variation diminishing (TVD) scheme in the LaxFriedrich formulation for the description of magnetic compression and diffusion in a cylindrical multidimensional geometry is the most suitable and is used in our model. Several models are being developed and can be used to calculate opacity: a collisional radiation equilibrium (CRE) model, which is a self-consistent model that takes into account Auger processes, and a nonstationary kinetic model that depends on the complexity of the problem and the availability of computer time. Radiation transport for both continuum and lines with detailed spectral profiles is being taken into account. The developed models are integrated into the HEIGHTS-EUV computer simulation package [1]. The package can also be used to study the hydrodynamics and radiation in two-gas mixtures of dense plasma focus (DPF) and z-pinch devices in the presence of impurities and erosion products that can affect radiation output.

In this paper, we discuss our model simulation of the MHD and optical processes that occur in various DPP devices of different electrode geometries. The general types of the DPP devices which can be simulated within the HEIGHTSEUV package are schematically shown in Figure 1: a) A dense plasma focus device, b) A hollow cathode triggered pinch plasma source, and c) A Z-pinch device [1-4]. The electrodes are drawn solid and shown in grey color. The device is filled by xenon gas under an initial pressure in the range of several tens of $m$ torr at room temperature, corresponding to an initial density of the gas in the range of $10^{14}-10^{15} \mathrm{~cm}^{-3}$. We assume that a preionization step that heats the gas to a temperature of near $1 \mathrm{eV}$ initiates the location of the discharge.

*hassanein@anl.gov; phone 1 (630) 252-5889; fax 1 (630) 252-3250

1. Presented at the SPIE Microlithography 2004 Conference, Santa Clara, CA, Feb. 22-27, 2004

Work supported partly by the Intel Corporation and International Sematech 


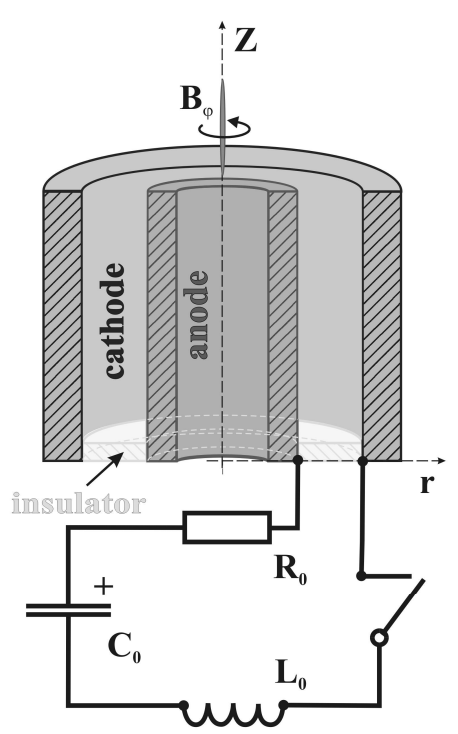

a) Dense Plasma Focus

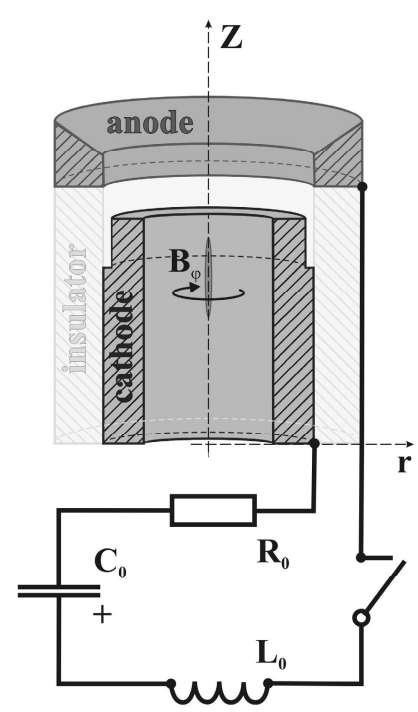

b) Hollow Cathode Tube

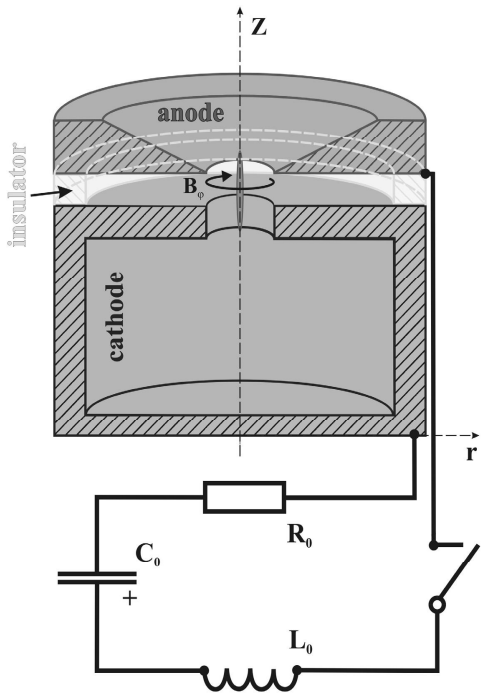

c) Z-pinch Device

Figure 1: Various configurations of DPP devices

The named devices are the conduction type i.e., the plasma is an element of the electric circuit, which gets the discharging current from the feeding capacitor $C_{0}$. The physical processes, which take place in these DPP devices are similar and can be described within the same mathematical model. Nonetheless, the important features of each particular design can also be reflected by the initial and boundary conditions, such as the design and geometry of solid walls, the initial plasma parameters, the parameters of the electric circuit, and the condition of turning the device off. The MHD, radiation transport, and thermal conduction are implemented as a kernel part of the HEIGHTS-EUV package, while design specific features are implemented as additional blocks.

\section{MATHEMATICAL MODEL}

The behavior of a conductive flow in the magnetic field due to current displacement triggered by the discharge is described by the general set of three-dimensional (3-D) resistive conservative MHD equations, including the processes of magnetic diffusion, thermal conduction, and radiation energy loss. The plasma is presented by the conservative variables of mass density $\rho$, momentum density $\rho \mathbf{v}$, total energy density $e$, and magnetic field $\mathbf{B}$. Details of implementing and solving the MHD equations by the TVD method in the Lax-Friedrich formulation for cylindrical geometries can be found elsewhere $[1,5,6]$. If not mentioned otherwise, all values in the paper are given in gaussian units.

In modeling actual DPP devices, such as Z-pinch, plasma focus, and other pinch types, the organization of the correct energy exchange is a key to successful simulation. The energy exchange is determined by a set of energy transport processes, such as thermal conduction and the radiation transport. The thermal conduction $\lambda$ is required to account for the gas-plasma media, the properties of which can significantly vary from point to point. Direct integration of the heat flux divergence,

$$
Q_{t h}=\frac{\partial e_{\mathrm{int}}}{\partial t}=\nabla \lambda \nabla T
$$

within the hydrodynamic algorithm can often lead to disconvergence of the solution. An alternative approach is to present the divergence term as a heat source and organize, in cylindrical coordinates, a separate numerical scheme: 


$$
\frac{\partial \mathrm{e}}{\partial \mathrm{t}}-\frac{1}{\mathrm{r}} \frac{\partial}{\partial \mathrm{r}} \mathrm{r} \lambda \frac{\partial \mathrm{T}}{\partial \mathrm{r}}-\frac{\partial}{\partial \mathrm{z}} \lambda \frac{\partial \mathrm{T}}{\partial \mathrm{z}}=0
$$

Finally, the heat source $Q_{t h}$ is the energy correction to the energy equation of the general MHD set:

$$
\frac{\partial e_{t o t}}{\partial t}+\nabla \cdot\left[\mathbf{v}\left(e_{t o t}+p_{t o t}\right)-\frac{1}{4 \pi}(\mathbf{v} \cdot \mathbf{B}) \mathbf{B}+\frac{c^{2} \eta}{16 \pi^{2}}(\nabla \times \mathbf{B}) \times \mathbf{B}\right]=Q_{t h}+Q_{r a d},
$$

where $\eta$ is specific plasma resistance. As previously described, to complete the system of MHD equations, thermodynamic pressure $p_{h}=p_{h}\left(e_{\text {int }}, \rho\right)$ and resistivity $\eta=\eta\left(e_{\text {int }}, \rho\right)$ functions are calculated from the equation of state [1]. Total energy density $e_{\text {int }}$ is a sum of internal, kinetic, and magnetic energy densities, and total pressure $p_{\text {tot }}$ is a sum of hydrodynamic and magnetic pressures.

During the evolution of the plasma pinch, the temperatures of the plasma can vary from parts of an eV to hundreds of $\mathrm{eV}$. At such different temperatures, several mechanisms can contribute to thermal conductivity. At low temperatures, when the ionization is absent, the classical elastic dispersion on neutrals mainly contributes to the total thermal conductivity. At low ionization states, an ambipolar diffusion, or diffusion of the neutrals in plasma, contributes to the total value. When a neutral appears in the hot area, it is ionized, and loses energy equal to the ionization energy. At higher ionization states, the electronic thermal conduction is a main contributor, which is given by the classic Spitzer formula with the correction of a non-ideal plasma. The process of energy transfer by the photons, emitted from the hotter areas and absorbed by the colder ones, is included directly in the form of radiation transport source, as described below.

The design features and the conditions of a particular EUV source are described in the form of boundary conditions [1, 7]. In simplified form, one may subdivide these conditions into three major parts. Hydrodynamic conditions are set on the boundaries of the hydrodynamic domain and include the conditions of the current flow, incoming /outgoing gas flow, and the symmetry of the device. Magnetic boundary conditions include the condition of the driven current in the electric circuit at the area of the supply voltage, the condition at the conductive walls, and the condition at the axes of symmetry to provide the cylindrical symmetry of the magnetic field. The thermal conduction conditions are set at the boundaries of the domain and include the condition of the given temperature of the wall, the isolative wall boundaries, and the income/outcome heat flux.

One of the critical mechanisms of initiating and governing the plasma discharge in a DPP device is the design and parameters of the external electric circuit. As shown in Fig, the electric circuit is normally defined by the capacitance $C_{0}$, resistivity of the wires $R_{0}$, and parasitical inductivity $L_{0}$. The circuit is incorporated into the MHD model, where the DPP device is given by its resistivity $R_{D P P}$ and inductitvity $L_{D P P}$. As far as the plasma parameters depend upon the whole dynamics of the discharge, they provide a feedback from the MHD to the electric circuit, changing the behavior of the current as a function of time.

The voltage drop at the DPP device is caused by the induced and the ohmic voltages. The inductance of the DPP device can be expressed by the Faraday law, which describes an induced voltage caused by the change of the magnetic flux. The resistance losses of the device are calculated from joule heating. Finally, the distribution of the continuous current in the electric circuit is regulated by the Kirchhoff law, which can be written for the voltage of a capacitor as a function of time. Being incorporated into the general set, the electric circuit equation is solved self-consistently with the MHD in the form

$$
\begin{aligned}
& U(t)=\frac{\partial}{\partial t} L_{0} I(t)+R_{0} I(t)+\frac{\partial}{\partial t} L_{D P P} I(t)+R_{D P P} I(t) \\
& \frac{\partial U(t)}{\partial t}=-\frac{I(t)}{C_{0}}
\end{aligned}
$$


where $I$ is the current and $U$ is the voltage. HEIGHTS simulations results of the electric current time evolution during the discharge are in excellent agreement with various DPP devices.

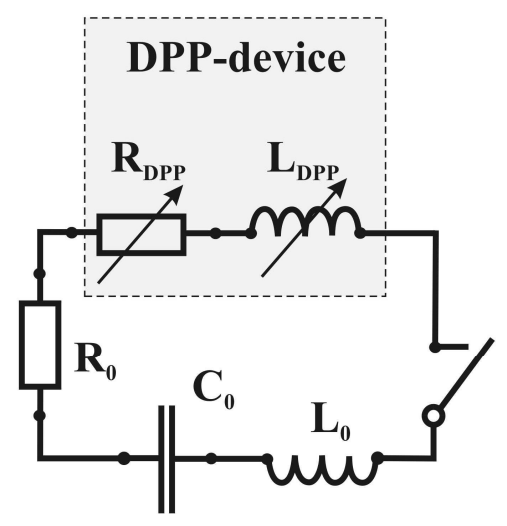

Figure 2: Schematics of an electric circuit for a typical DPP device

\section{RADIATION TRANSPORT}

In studying the transfer of radiant energy, one needs to distinguish two aspects in modeling a DPP device. First, in solving the hydrodynamic part of the problem, the redistribution of the plasma internal energy must be corrected, as well as the evolution of the whole pinch dynamics. Key is the accurate and detailed calculation of the radiant energy redistribution in the whole plasma domain within the very broad spectral range of participating photons. The second aspect is the detailed calculation of the effective radiation of the EUV source within the operating $13.5 \pm 2 \% \mathrm{~nm}$ spectral energy range. In this case, the radiation transport must be calculated to account for those photons whose energies are in the mentioned range.

In numerical simulation of the radiation transport processes, the radiant energy flux is calculated by integrating the equations of the radiation transport (RTE) [8,9], which represents the conservation of emitted and absorbed energy along the direction of the photon transfer. Deterministic transport methods discretise the calculation domain in space, energy, and angles [10]. The radiation intensity along each direction is determined by integrating the RTE, and the directions are given by the angles of the chosen numerical quadrature. A detailed description of the method, as well as its numerical implementation and obtained results, are presented in [1,6]. Nevertheless, conducting parametric studies of a DPP device is difficult because this method is time-consuming and resource intensive. Recognizing the need to accurately calculate the radiation transport in a reasonable amount of time, and to ensure the validity of our simulation, we have developed and implemented an alternative method for calculating the radiation transport by means of MonteCarlo imitation modeling [11].

In contrast to the direct resolution of the integral-differential RTE, where one searches the continuous function of the radiation intensity, the probabilistic model of the energy redistribution operates with discrete portions of the radiative energies. Each energy portion is individually analyzed. Studying the trajectory and the behavior as well as the number of emitted and absorbed test energy portions in each point of the plasma domain (or in the points which represent the major interest), one may re-distribute the energy due to photon transport. The energy and the trajectory of photon movement are determined by the Monte-Carlo techniques. The total energy of a portion is a sum of the energies of the component photons, and the portion is called a "macro-photon" or "photon bundle."

Depending upon the hydrodynamic parameters of a cell, a selected number of probe macro-photons is analyzed by the Monte-Carlo radiation transport method (MCRT). The energies of the macro-photons are distributed accordingly to the emission coefficient of the cell. Later on, the history, the behavior, and the track of the photons are statistically accumulated in the form of energy redistribution within the domain. 
To calculate the number of photons $N$, emitted within the spectral range $\left[E_{\min }, E_{\max }\right]$ by the domain of the volume $V$ at unit time, the emission coefficient $\kappa_{\text {emi }}(T, \rho, \varepsilon)$ needs to be integrated with the Planck function over the total spectrum of energies $\mathcal{E}$ [12]:

$$
N=V \int_{E_{\min }}^{E_{\max }} \frac{\kappa_{\mathrm{emi}}(T, \rho, \varepsilon) \varepsilon^{2}}{\hbar^{3} \pi^{2} c^{2}}\left(e^{\frac{\varepsilon}{T}}-1\right)^{-1} d \varepsilon, \mathrm{cm}^{-3} \mathrm{~s}^{-1} .
$$

Here, as elsewhere below, we use the traditional notation for the Planck constant $\hbar$ and speed of light $c$. The energy of the photon is calculated from the absorption probability density, which is expressed through the emission of the cell. After moving through the domain, the photon can be absorbed as described by the absorption probability density, which exponentially grows with the photon track length. Finally, one derives the correction $Q_{\text {rad }}$ in the energy equation (3) due to radiation transport, knowing the number and the energy of emitted and absorbed photons in each cell of the hydrodynamic domain:

$$
Q_{\text {rad }}=\sum_{i}^{N_{a b s}} \varepsilon_{i}-\sum_{j}^{N_{e m i}} \varepsilon_{j} .
$$

The efficiency of the simulation is significantly improved by introducing a set of weight factors. Based on the emission of the cells, the first factor is used to rebalance the number of emitted photons from the less-emitting cells in favor of the most-emitting ones. The accuracy of the computation is regulated by the maximal number $N_{\max }$ of photons emitted from a cell. Another weight factor is used to avoid following those photons, which are emitted and immediately absorbed within the same cell.

The comparative results of the accuracy for discrete ordinates and Monte-Carlo radiation transport methods are shown in Fig. 3 for typical temperature and density distributions, obtained from the simulation of a DPP device. The quantitative and qualitative behavior of the radiation flux are the same, despite the Monte-Carlo method being significantly faster by approximately a factor of more than two orders of magnitude! This also confirms the high accuracy and reliability of the implementation of our methods.
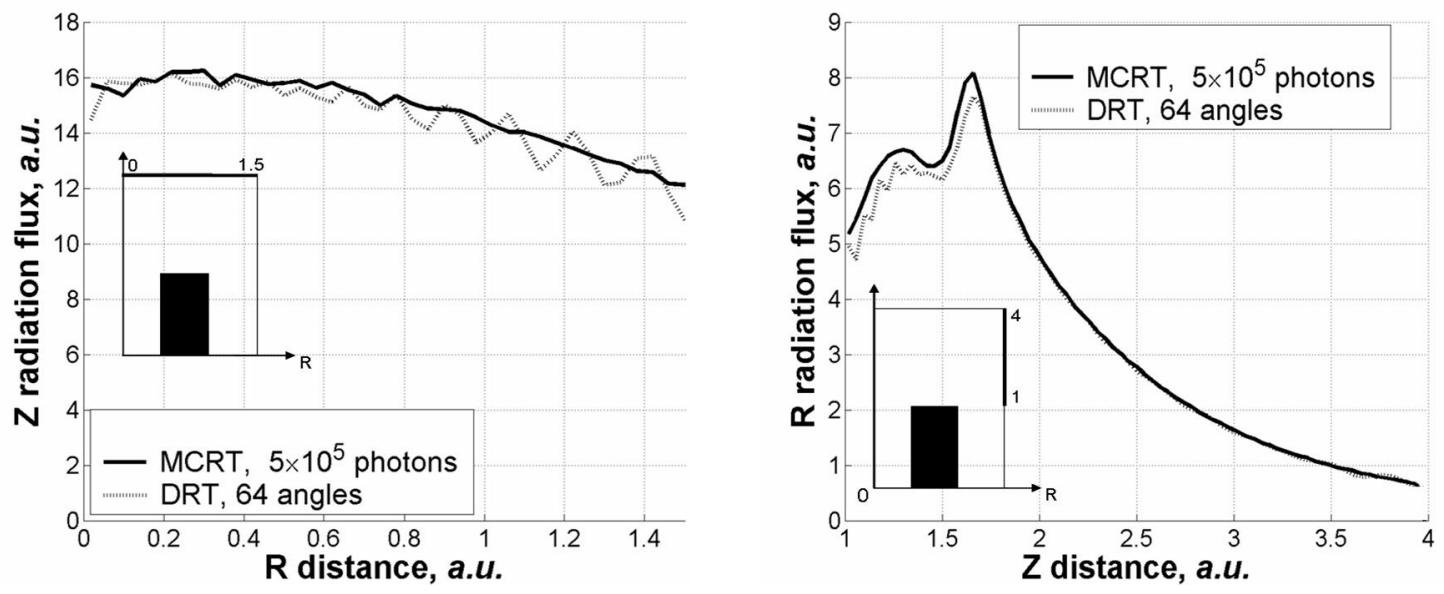

Figure 3: Comparison of different methods of HEIGHTS-EUV radiation transport 


\section{OPACITIES}

In the computer calculation of radiation transport in plasma, the integral radiation fluxes depend to a great extent upon the level of detail and the precision of the optical coefficients. In their turn, the computational accuracy and completeness of calculated opacities depend upon the accuracy and completeness of the atomic data (such as electronic wave functions, energy levels, transition probabilities, oscillator strengths, and other derived values) and populations of atomic levels. Nowadays, such self-consistent field methods as Dirac-Fock (DF) or Hartree-Fock (HF) methods are believed to be the most accurate and appropriate for practice $[13,14]$.

The simulation of the dynamics of the pinch evolution is a typical problem that involves a wide range of temperature and density values with a very complicated ionic structure. Detailed resolution of each possible level for each possible ion, (and consequently, each electronic transition in the ion) in a wide range of temperatures, densities, and energies is enormously laborious work, especially when one is accounting for the complicated hyperfine structure of each split level. That is why, in consistently solving the MHD equations and the RTE, the opacities are obtained based on simplified methods of calculation for the structures of atomic levels and transition probabilities. One such method is the Hartree-Fock-Slater (HFS) approximation [15], which allows the determination of the energies and other atomic characteristics for each $n l$-configuration of each ion that might exist in the plasma. Normally, the shift of some spectral lines from their true places by several percent is not problematic in determining the integral radiation flux. In the MHD calculation this permits utilization of the opacities calculated on the basis of the HFS methods with splitting of the energy levels and spectral lines within the framework of the perturbation theory.

As shown earlier in $[1,6,16,17]$, accounting for electrostatic and spin-orbit splitting of configurations and spectral lines considerably influences the dynamics of the energy balance in the plasma. A method of calculating the optical coefficients for high-Z plasma was developed and implemented in [16] by means of the collision-radiation equilibrium (CRE) model. The important feature of this method is the joint use of HFS atomic data and Racah techniques of angular moments. This feature makes possible the use of comparatively unsophisticated methods to consider the complex electronic structure of each participating ion, and the complicated splitting of each configuration into mathematical terms (using the LS-coupling approximation), over a wide range of spectral frequencies, and in the expected range of temperatures and densities. By this technique, the detailed emission and absorption spectra are initially calculated over a very complete spectral frequency scale (up to 100,000 points) for the expected range of MHD values. The width of the frequency interval was comparable to the Doppler width of the strongest spectral lines, which provides a satisfactory resolution of the line profiles. Because of the large size of the generated opacity tables, the practical use of such detailed data is not convenient, and the emission and absorption coefficients are thus averaged in spectral groups.

A rigorous theory for averaging the opacities within a group of frequencies does not exist. Such an averaging procedure is considered correct only when the absorption coefficient is basically constant within the group, or the optical thickness of each line is very small, and the absorption becomes a linear-like function from the frequency. This situation is only possible for a continuum, but even in that case, every photo-ionization threshold must become a boundary of a group. The situation becomes more complicated for the lined spectrum, when the absorption coefficient often drops several orders of magnitude within a very limited frequency interval, say, from the center of a very strong spectral line to the wings of the same line, and the center of the line is optically thin. Moreover, the temperature and density values may vary. This change leads to spectral lines for the other ions, and the width of the existing lines is also changed.

From a practical viewpoint, an organized selection of the strongest lines is a reasonable way to describe the optical coefficients within the most important hydrodynamic areas for typical temperature and density values. The other lines are averaged within broad groups. Unfortunately, the primary goal of the numerical simulation is the determination of the typical hydrodynamic parameters within the important areas of the plasma domain!

For a uniform isothermal plasma, the optical thickness of a spectral line is determined by multiplying the absorption coefficient of the line by the linear dimension of the plasma $\tau(\varepsilon)=\kappa_{\text {abs }}(\varepsilon) \cdot l$. In the case of a nonuniform nonisothermal plasma, this definition is generalized as $\tau(\varepsilon)=\int \kappa_{\text {abs }}(T, \rho, \varepsilon) d l$ over the interval $\Delta l$, where the ion exits emitting with frequency $\mathcal{E}$. 
The borders of the groups are calculated from the following considerations. First, the width of a group $\Delta \varepsilon$ should never exceed $\alpha T$, where $T$ is the plasma temperature, and $\alpha$ is a chosen parameter for averaging. This condition provides the smooth averaging of opacities in the continuum region, where the averaging is normally performed within the broad groups, and the optical thickness is much less than one. Second, invariability of the optical coefficient is required for a chosen value $\beta$ within the group. This condition provides a very specified resolution of the spectral lines with the optical thickness nearly at unity, and the wings of the lines with the optical thickness greater than unity. A final consideration is that, all those frequencies which belong in the detailed spectrum to a line with $\tau(\varepsilon) \geq 1$ are also included in the domain of the groups. This condition provides a very thorough resolution of the strong lines in the averaging spectrum. By variation of the parameters $\alpha$ and $\beta$, several group mean opacities are generated with different levels of completeness and detail.

Based upon several recent studies [3,18], it is supposed that the maximal radiation transport corresponds to the moment of pinch formation. A typical pinch parameters are the following: the temperature is close to $25 \mathrm{eV}$, the density is $10^{17} \mathrm{~cm}^{-3}$, the spectral range in radiation energies varies from $5 \mathrm{eV}$ to $250 \mathrm{eV}$, and the average optical plasma thickness is $1 \mathrm{~cm}$. We have generated two sets of opacities, averaged within 240 and 791 spectral groups. A comparison of the radiation fluxes, has shown the convergence of the fluxes, and allows one to pick out the values of hydrodynamic parameters from the most emitting areas.

Taking a wider set of temperature and density pairs we have generated the optimal scale of spectral groups, after which, combining all scales, the resulting energy scale has a total of 3639 energy groups. The result of the self-consistent hydrodynamic/radiation transport calculations with these opacities can also be used for further improvements in the quality of the coefficients. We have calculated and compared the dynamics of the typical DPP device with the opacities averaged over both 791 and 3639 groups. The dynamic of the plasma is left unchanged, signifying that 791 groups is a quite reasonable initial choice. Nevertheless, during the time of formation and evolution of the pinch, the detailed opacities result in more accurate values within the 5\% range. Such a difference is quite insignificant from the hydrodynamic point of view, but, as seen from Fig. 4, important from the point of view of collectable EUV energy.

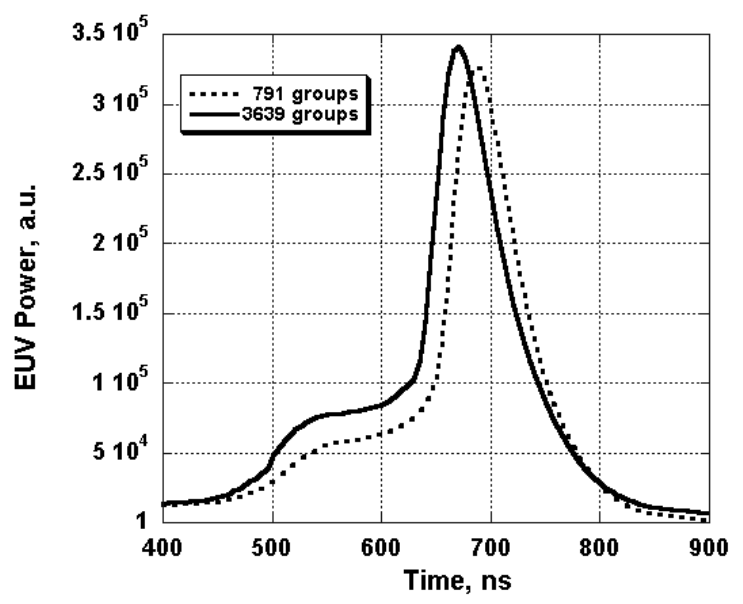

Figure 4: Typical pinch temperature and density values, 791 vs. 3639 optical groups.

In the first part of modeling, the radiation transport is calculated self-consistently for the magnetic hydrodynamics, as described above. The goal is to obtain the temperature and density distributions needed for the second part of modeling: the calculation of the EUV power of the source. The second part utilizes the same MCRT method, but using another set of opacities.

In using the HFS model for calculation of energy levels, the typical accuracy of the result is within 3-5\%; this is inappropriate for the EUV range of $13.5 \pm 2 \%$. Compared to HFS, the more accurate HF model treats the upper and lower states split into components, and each component is a set of levels with different quantum numbers $L, S$, and $J$. 
The values of splitting can exceed the required $2 \%$ of the transition energy. It becomes important to know which fraction of the whole transition is situated within the range of interest. One should also remember that there are several well-known HF methods, and their implementation, each of which calculates the energy levels (and transitions) slightly different, can also be important for such a narrow range [19].

In calculation of a lower state, the average term is often approximated, when all states with different $L, S$, and $J$ quantum numbers are supposed to have the same radial wave function and eigenvalue. The splitting by $L, S$, and $J$ is defined by Slater integrals and various angular factors, which represent electrostatic, spin-orbit, and exchange interactions. Relativistic corrections are found within the limits of the perturbation theory. Being mathematically stricter, the $L S D$ HF method is used to calculate the total energy of a term by solving a separate set of HF equations for each ${ }^{S} L$ term [19]. The method should be called $L S v D$ HF, because the total energy for $d$-shells also depends on the seniority number $v$.

As previously shown [16], the details and the accuracy of calculating energy levels depend mainly on the method of implementing the $J$-splitting. The simplest way to account for splitting within the first order of the perturbation theory is to use the diagonal Lande correction [16,19]. Nevertheless, xenon ions are characterized by the intermediate coupling, when electrostatic and spin-orbit splittings are comparable.

The widely used method of calculating the energy of the upper state is to take the difference of the eigenvalues of the wave functions in the ground and excited states, accounting for splitting of the ground state within the average term approximation. Accounting for splitting of the upper state is more accurate, but the corrections should be added to the difference, which produces a very large number of transition components, only part of which can fall into the EUV range. The very accurate method is the calculation of upper and lower states in intermediate coupling for two open shells.

Generalizing, despite the variety of approaches, $L S v D$ HF in intermediate coupling was chosen as the most appropriate and most accurate method for both upper and lower states. The calculation was performed for $4 d^{n}-4 d^{n-1} 4 f, 4 d^{n}-4 d^{n 1}-5 p$, and $4 p^{6} 4 d^{n}-4 p^{5} 4 d^{n+1}$ transitions of Xe IX - Xe XVIII ions.

\section{RESULTS AND DISCUSSION}

The results of a DPP discharge depend on the detailed physics of the discharge, the geometrical design of the device, the electric circuit design, the initial parameters in the chamber, and the current profile. As simulated by the HEIGHTSEUV package, we present the comparative results of detailed simulation of the discharge near the moment of plasma pinch for the typical designs of the EUV sources. The measurements of the pinch, provided in [20], are in very good agreement to the HEIGHTS simulation, as seen from Fig. 5. Similar measurements of the pinch, presented by other source developers, are in very good agreement with HEIGHTS.
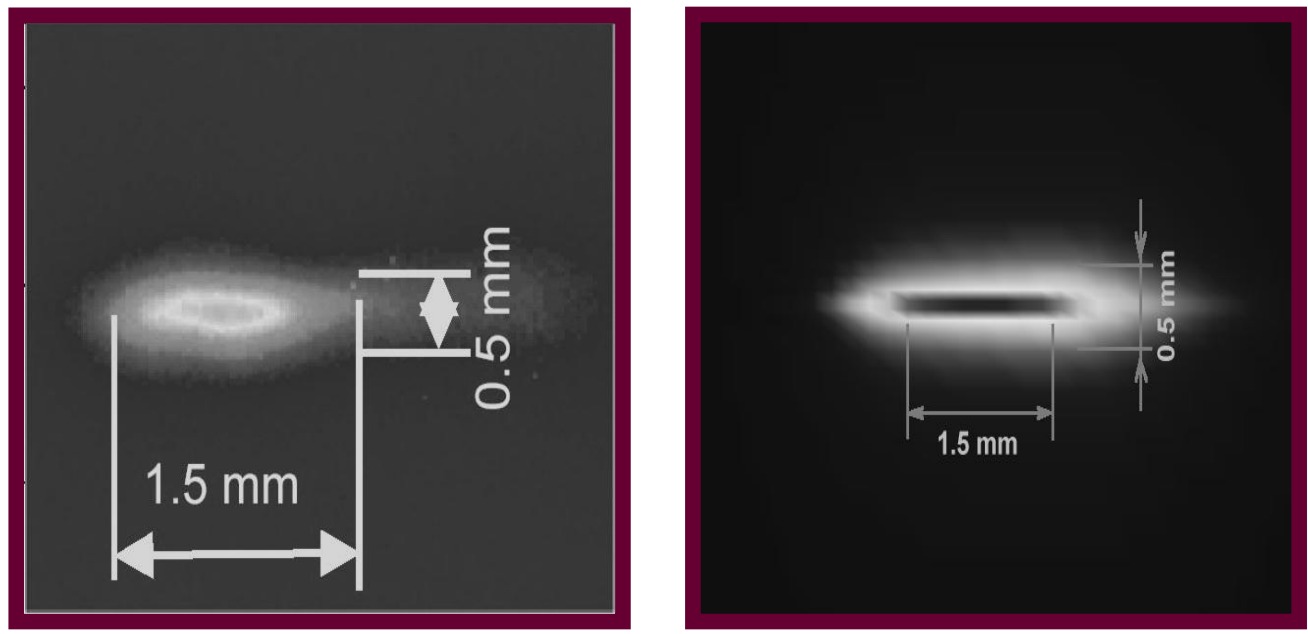

Figure 5: Details of the pinch, experimental study (left) [20] and HEIGHTS-EUV simulation (right) 
In optimizing the design of a DPP device, the clear advantage of using the computer simulation is its flexibility for changing the critical parameters of the discharge. As well known and noted in various studies $[1,20,21]$, the electric circuit parameters are the main ones and vital to the whole dynamics of the pinch in all terms, in pinch time, reaching the desired thermodynamic state, and the amount of collectable EUV. The increase of the electric energy input of the pinch plasma source triggered by a hollow cathode and for given initial conditions in the chamber would lead to a clear maximum in collectable EUV energy. However, a similar input energy increase in a Z-pinch device gives different maximum value for the EUV output energy.

\section{CONCLUSIONS}

This paper presents an integrated model that is being developed to describe the hydrodynamics and optical processes that occur in DPP devices. The model addresses three main subjects: plasma evolution and MHD processes, detailed photon radiation transport, and interaction between plasma/radiation and material. The developed models and numerical methods are being implemented and integrated in the HEIGHTS-EUV computer simulation package. This package, will be a tool that can be used to study, optimize, and enhance our understanding of the hydrodynamics and radiation processes in dense plasma discharge devices and the issues related to component erosion and lifetime estimates.

\section{REFERENCES}

1. A. Hassanein, V. Sizyuk, V. Tolkach, V. Morozov, and B. Rice, HEIGHTS initial simulation of discharge produced plasma hydrodynamics and radiation transport for EUV lithography, Proc. of SPIE Vol. 5037, pp. 714-727, 2003.

2. W. Partlo, et al., Development of an EUV $(13.5 \mathrm{~nm})$ light source employing a dense plasma focus in lithium vapor, The Source 31, 3, 2001.

3. K. Bergman, et al., Highly repetitive, extreme-ultraviolet radiation source based on a gas-discharge plasma, Appl. Opt., Vol. 38, No. 25, pp. 5413-5417, 1999.

4. U. Stamm, Gas discharge and laser produced plasma sources at XTREME technologies, International SEMATECH EUV Source Workshop, Feb. 23, 2003, Santa Clara, CA, USA.

5. Gábor Tóth, The $\nabla \cdot \mathrm{B}=0$ constraint in shock-capturing magnetohydrodynamics codes, J. Comp. Phys., Vol. 161, pp. 605-652, 2000.

6. A. Hassanein, V. Sizyuk, V. Tolkach, V. Morozov, and B. Rice, HEIGHTS initial simulation of discharge produced plasma hydrodynamics and radiation transport for extreme ultraviolet lithography, accepted for publication at Journal of Micromechanics and Microengineering, Jan. 2004.

7. O. Diyankov et al., MAG - two-dimensional resistive MHD code using an arbitrary moving coordinate system, Comp. Phys. Comm. Vol. 106, pp. 76-94, 1997

8. R. Siegel and J. R. Howell, Thermal Radiation Heat Transfer,3rd ed., Hemisphere, Washington, DC, 1994.

9. B. G. Carlson, Solution of the Transport Equation by $S_{n}$ Approximations, Los Alamos National Laboratory, Report LA-1599, 1953.

10. E. E. Lewis, and W. F. Miller Jr, Computational Methods of Neutron Transport, Wiley-Interscience, New York, 1984.

11. R. Siegel and J. R. Howell, Thermal Radiation Heat Transfer, Hemisphere Publishing Corporation, Washington DC, p. 862, 1981.

12. Ya. B. Zeldovich and Yu. P. Raizer, Physics of Shock Waves and High-Temperature Hydrodynamics Phenomena, Vol. 1, Academic Press, New York, p. 464, 1966.

13. H. A. Bethe, Intermediate Quantum Mechanics, Walter Benjamin, New York, p. 276, 1964.

14. F. Herman and S. Skillman, Atomic Structure Calculations, Prentice-Hall, Englewood Cliffs, NJ, 1963.

15. J. C. Slater, A simplification of the Hartree-Fock method, Phys. Rev.Vol. 81, p. 385, 1951.

16. V. Tolkach, A. Hassanein, and V. Morozov, Development of Comprehensive Models for Opacities and Radiation Transport for IFE System, Argonne National Laboratory, Report ANL-ET/02-23, 2002.

17. H. Würz et al., Fusion Sci. Technol. Vol. 40, No. 3, pp. 191-246, 2001.

18. E.R. Kieft., et al., Time-resolved pinhole camera imaging and extreme ultraviolet spectrometry on a hollow cathode discharge in xenon, Phys. Rev. E, Vol. 68, 056403, 2003.

19. R.D. Cowan, The Theory of Atomic Structure and Spectra, Univ. of California Press, Berkeley, 1981. 
20. XTREME Technologies, Performance update high power gas discharge EUV source development, Presented at ISMT EUV Source Workshop, Antwerp, Belgium, Sept. 29, 2003.

21. G. Derra and T. Krücker, Modeling of EUV sources - What can we expect from it? Presented at Sematech EUV Source Modeling Workshop, Antwerp, Belgium, Sept. 28, 2003. 